CAHIERS DE

NARRATOLOGIE

\section{Cahiers de Narratologie}

Analyse et théorie narratives

32 | 2017

Récit et argumentation, interactions, lieux et dispositifs sociaux

\title{
La métalepse du lecteur Ou la porosité du métatexte
}

\section{Bertrand Daunay}

\section{(2) OpenEdition \\ Journals}

Electronic version

URL: http://journals.openedition.org/narratologie/7855

DOI: $10.4000 /$ narratologie. 7855

ISSN: 1765-307X

Publisher

LIRCES

Electronic reference

Bertrand Daunay, "La métalepse du lecteur Ou la porosité du métatexte », Cahiers de Narratologie [Online], 32 | 2017, Online since 21 December 2017, connection on 14 November 2019. URL : http:// journals.openedition.org/narratologie/7855; DOI : 10.4000/narratologie.7855

This text was automatically generated on 14 November 2019.

Article L.111-1 du Code de la propriété intellectuelle. 


\title{
La métalepse du lecteur Ou la porosité du métatexte
}

\author{
Bertrand Daunay
}

\section{Introduction}

1 «Qui a tué Roger Ackroyd? » On se rappelle la question dont Pierre Bayard (1998/2002) faisait le titre d'un ouvrage où il voulait, disait-il, « réfléchir sur la nature ou les fondements d'une lecture vraie» par «l'édification expérimentale d'une lecture délirante» (p. 15). Une telle question, qui pastiche le genre immémorial des questions de compréhension en contexte scolaire, mime une caractéristique bien connue et en même temps bien étrange du métatexte ordinaire qui prend pour objet de discours un texte de fiction: une forme de confusion énonciative entre le (monde du) texte et le (monde du) métatexte, entre (récit) fictif et (commentaire) réel. "Roger Ackroyd", personnage de fiction, peut ainsi intégrer un métatexte et faire l'objet d'un discours réel, le métatexte redoublant l'effet propre de la fiction qui est précisément de faire comme si les évènements narrés étaient réels. Il s'agit certes d'une façon de parler, d'une figure : mais de la figure à la fiction, pour emprunter l'expression et l'idée au soustitre d'un ouvrage de Genette sur la métalepse (2004), il n'y a finalement qu'un élargissement.

2 C'est cette manière dont un métatexte, par une figure, emprunte à la fiction, que je voudrais interroger ici. Et ma référence à la métalepse s'explique par le fait que je vois, dans la (con)fusion du texte et du métatexte, une transgression de même nature que celle qu'opère ce que Genette appelle la métalepse de l'auteur (1972, p. $244 ; 2004$, p. 10). À cette métalepse, qui donne à l'auteur (réel) le moyen de feindre de briser la frontière de l'extradiégétique et du diégétique, me semble correspondre une figure parallèle, qui permet au lecteur (réel) de feindre une telle transgression des frontières : j'appellerai métalepse du lecteur cette figure par laquelle le scripteur d'un métatexte (con)fond, dans son discours, le réel et le fictif, en feignant de croire que l'on peut réellement se demander, en dehors de la fiction, qui a tué Roger Ackroyd. 
3 Cette figure, assurément banale, est nécessaire à l'élaboration même du discours métatextuel et se révèle finalement le signe du ressort de toute lecture, qui consiste à créer un lien entre le monde du texte et celui du lecteur. Mais dans sa banalité, cette figure ne manque pas d'étonner quand on s'y arrête, si du moins l'on a opté pour une frontière entre fait et fiction - je paraphrase par ces mots le titre de l'ouvrage récent de Françoise Lavocat (2016), qui n'ignore pas mais déjoue la résurgence postmoderne du topos de la porosité de cette frontière. Et je la suis dans sa démonstration comme dans ses conclusions : défendre des frontières « ne signifie pas que l'on s'y claquemure » et permet au contraire de prendre acte « des multiples façons de les passer ou de croire les passer » (ibid., p. 521 sq.). Lavocat identifie la métalepse de l'auteur comme l'une de ces façons de franchir (fictivement) les frontières entre fait et fiction; la métalepse du lecteur me semble en être une autre, qui mérite d'être analysée.

Or il me semble que le phénomène de la métalepse du lecteur a été peu décrit, malgré quelques propositions théoriques intéressantes ces dernières années, et je me propose de reprendre à nouveaux frais ce dossier ${ }^{1}$. Je n'entrerai pas ici dans le détail de la description des manières dont se réalise une métalepse du lecteur ${ }^{2}$, mais je me propose d'avancer quelques réflexions théoriques afin de cerner ce concept de métalepse du lecteur (première partie), pour aboutir (deuxième partie) à une double extension, en montrant que la métalepse du lecteur est finalement liée à toute lecture de textes de fiction et n'est pas sans relation avec certaines caractéristiques de tout discours métatextuel ${ }^{3}$.

\section{De la métalepse de l'auteur à la métalepse du lecteur : questions de frontières}

5 Pour penser la métalepse du lecteur, je propose un petit détour historique par la métalepse de l'auteur et sa construction conceptuelle, afin d'identifier les frontières en jeu et leurs possibles transgressions métaleptiques.

\section{La métalepse de l'auteur et du narrateur}

6 On se rappelle que Genette définit la métalepse en trois petites pages de Figures III (1972, p. 243-246). Il identifie en premier lieu des métalepses «aussi banales et innocentes que celles de la rhétorique classique » (p. 244), qui ne font que (con)fondre deux temporalités, celle de l'histoire racontée et celle de la narration, comme dans cet exemple, qu'il prend à La Recherche du temps perdu :

Je me contente ici, au fur et à mesure que le tortillard s'arrête et que l'employé crie Doncières, Grattevast, Maineville, etc., de noter ce que la petite plage ou la garnison m'évoquent.

7 Genette évoque ensuite des exemples de métalepses " plus hardis, c'est-à-dire un peu plus littéraux» (p. 245), citant Sterne, Pirandello, Genet, Robbe-Grillet - et l'on peut ajouter l'exemple qu'il avait précédemment donné (p. 244) de la nouvelle de Cortázar, «Continuité des parcs », où un homme est assassiné par un personnage du roman qu'il lit.

8 Si Genette voit des degrés de «hardiesse ${ }^{4}$ » ou de «littéralité », il n'établit pas de différence de nature entre toutes les métalepses qu'il évoque. On peut cependant, à la 
suite de Marie-Laure Ryan (2005, p. 205-209), distinguer les métalepses rhétoriques simples jeux discursifs, sans réel basculement des univers diégétique et extradiégétique (comme dans l'exemple tiré de La Recherche) - et les métalepses ontologiques, où les entités de ces deux univers passent de l'un à l'autre (comme dans l'exemple de "Continuité des parcs ») .

Cette distinction ne doit pas faire oublier la continuité entre les deux, les métalepses ontologiques n'étant finalement qu'une extension des métalepses rhétoriques. Du reste, on se rappelle que, dans sa première approche de la métalepse, Genette évoque la "rhétorique classique », à laquelle il emprunte la figure, pour la reconstruire dans son propre cadre narratologique. Arrêtons-nous rapidement sur cette généalogie : Genette, pour présenter la métalepse, cite Pierre Fontanier et la description qu'il fait d'une forme particulière de métalepse, ce "tour par lequel un poète, un écrivain, est représenté ou se représente comme produisant lui-même ce qu'il ne fait, au fond, que raconter ou décrire » $(1821 / 1968$, p. 128). À l'appui, Fontanier cite ces premiers vers des Trois règnes de la nature de Delille :

Enfin j'arrive à toi, terre à jamais féconde,

Jadis de tes rochers j'aurais fait jaillir l'onde.

10 Voici le commentaire que fait Fontanier de ces vers (ibid.) : « Il veut dire qu'il aurait pu autrefois chanter l'eau jaillissant des rochers. » Fontanier (ibid., p. 129) « rapporte » à la métalepse une autre figure, un autre tour, celui par lequel « on abandonne tout à coup le rôle de narrateur pour celui de maitre ou d'arbitre souverain, en sorte que, au lieu de raconter simplement une chose qui se fait ou qui est faite, on commande, on ordonne qu'elle se fasse ». Pour exemple, ce vers de Voltaire (Poème de Fontenoy) :

Maison du roi, marchez, assurez la victoire.

11 Les deux exemples que donne Fontanier ${ }^{6}$ ont ceci de commun que l'écrivain feint d'agir sur l'objet qu'il invente, le narrateur crée au lieu de raconter le monde. La métalepse ou quand dire, c'est faire... Ce qui est en jeu ici est le brouillage des frontières, celle de la narration et du monde narré, qui recoupe celle du réel et de la fiction - et c'est précisément ce qui permet l'extension de la figure rhétorique à la narratologie, chaque fois que peut se percevoir une transgression des frontières narratives.

Or il se trouve que, en identifiant un brouillage de frontières, Fontanier, dans ses commentaires, en réalise une autre : celle qui sépare l'écrivain et le narrateur, les deux termes étant employés indifféremment par lui. Cette confusion, assez normale chez Fontanier, amène Genette à la faire aussi dans sa description de la métalepse ; décrivant la métalepse narrative en parlant au départ de métalepse d'auteur ${ }^{7}$, il conclut (1972, p. 245):

Le plus troublant de la métalepse est bien dans cette hypothèse inacceptable et insistante, que l'extradiégétique est peut-être toujours déjà diégétique, et que le narrateur et ses narrataires, c'est-à-dire vous et moi, appartenons peut-être encore à quelque récit.

13 Analysant la figure par laquelle est transgressée, à l'intérieur d'un récit, la frontière entre les niveaux narratifs, Genette réalise une figure de même nature, en transgressant la frontière entre niveaux textuels, confondant un monde représenté (celui du narrateur et du narrataire) et le monde réel (celui de vous et moi).

14 Pour être plus exact, il faudrait dire que Genette feint de transgresser une frontière qu'il pose lui-même par ailleurs (1972, p. 265) : 
Comme le narrateur, le narrataire est un des éléments de la situation narrative, et il se place nécessairement au même niveau diégétique; c'est-à-dire qu'il ne se confond pas plus à priori avec le lecteur (même virtuel) que le narrateur ne se confond nécessairement avec l'auteur.

Si l'on peut considérer ici, vu le jeu littéraire que constitue cette conclusion du passage de Figures III sur la métalepse (qui s'explique par la citation de Borges qui le précède), que c'est une fiction de transgression, il faut reconnaitre qu'à d'autres endroits, Genette ne feint pas. Ainsi, la première fois (sauf erreur) que Genette évoque la métalepse, dans Figures II (où il décrit le phénomène dans Moyse sauvé de Saint-Amant, à partir de la définition de Fontanier), il parle de «métalepse de l'auteur ou du narrateur » (1969, p. 216, n. 3). C'est aussi le cas, on l'a vu, au début du passage de Figures III où il décrit la métalepse, quand, à propos de Sterne, il passe indifféremment de l'auteur au narrateur ou du lecteur au narrataire (1972, p. 244). De même, quand il revient sur la métalepse, une dizaine d'années plus tard (1983, p. 58), il la définit comme ce qui arrive « lorsqu'un auteur (ou un lecteur) s'introduit dans l'action fictive de son récit ou lorsqu'un personnage de cette fiction vient s'immiscer dans l'existence extradiégétique de l'auteur ou du lecteur ». L'extradiégétique est un niveau narratif, pour Genette, qui relève de la fiction ${ }^{8}$ : ce ne sont donc pas l'auteur ou le lecteur, instances réelles, qui sont concernés, mais le narrateur ou le narrataire, instances fictives - même s'il est loisible au lecteur d'identifier le narrateur à l'auteur (quand rien ne vient donner corps à l'instance narrative) ou de s'identifier lui-même avec le narrataire (ce qui est plus aisé quand ce dernier est construit par le texte sans trait distinctif mais comme une sorte d' auditoire universel ${ }^{9}$ ).

Qu'il feigne ou non, quand Genette met sur le même plan narrateur et auteur, narrataire et lecteur, il brise une frontière entre réalité et fiction : il œuvre alors luimême dans la fiction et redouble ou instaure à nouveau, dans son métatexte, l'effet d'une illusion qu'élabore le texte de fiction. C'est cette transgression des niveaux qui me semble à son tour une métalepse: non pas une métalepse de l'auteur, mais une métalepse du lecteur cette fois.

\section{Jeux de frontières et métalepses}

17 La figure suivante représente ces jeux de frontières et les lieux où se jouent la métalepse de l'auteur et celle du lecteur - pour avancer dans notre réflexion, j'ajoute tout de suite dans cette figure une autre frontière : celle du texte et du métatexte ${ }^{10}$.

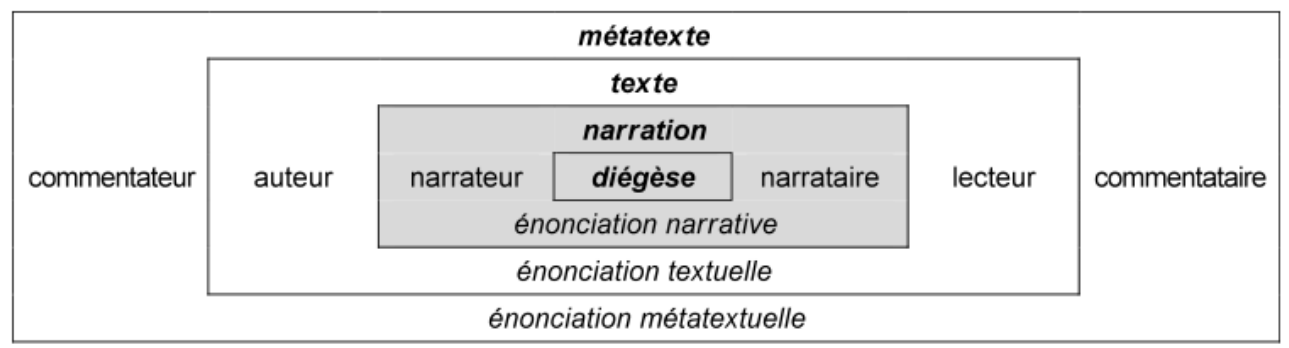

Figure 1. Frontières textuelles

Dans cette figure ${ }^{11}$, on peut identifier chaque cadre comme objet de discours du niveau supérieur: le texte est objet de discours du métatexte, la narration est l'objet de discours du texte, la diégèse est l'objet de discours de la narration, dans des situations d'énonciations différentes (que j'ai appelées énonciations métatextuelle, textuelle, 
narrative $\mathrm{e}^{12}$ ), prises en charge par des instances d'énonciation particulières (le commentateur, l'auteur, le narrateur), pour des destinataires spécifiques (le commentataire $^{13}$, le lecteur, le narrataire).

Il faudrait ajouter évidemment tous les récits enchâssés au sein de la diégèse, laquelle peut, à l'instar de poupées gigognes, contenir de nouveaux récits internes, aux niveaux que Genette appelle métadiégétiques ${ }^{14}$. Mais la question est assez complexe pour qu'on puisse se passer de ce niveau ici, qui n'importe que marginalement à notre propos.

Je propose de réserver ce que Genette appelle métalepse narrative ou métalepse $d u$ narrateur aux transgressions des frontières au sein de l'énonciation narrative, qui relève de la fiction (c'est la partie grisée du schéma); c'est le cas chaque fois qu'un narrateur intervient dans le récit qu'il narre ou que le narrataire intervient dans le récit narré (et rappelons que cette situation peut se reproduire au sein de la diégèse, quand une histoire y est racontée), ce qui n'est possible que fictivement (dans le monde fictif créé par l'auteur). Réservons alors l'expression métalepse de l'auteur au cas, que j'évoquais à l'instant, où l'auteur feint (par une figure) de pouvoir intervenir dans le monde fictif qu'il crée comme si c'était le même niveau de réalité (c'est le cas de la métalepse de la rhétorique classique) ou quand l'auteur feint de faire intervenir le lecteur réel dans son histoire, confondant narrateur et auteur, narrataire et lecteur : on pourrait aussi parler à ce sujet, de métalepse textuelle, qui se joue au niveau de l'énonciation textuelle.

21 Si de telles transgressions sont le fait non de l'auteur mais du lecteur, on glisse alors vers ce que j'appelle la métalepse du lecteur. Cette dernière pourrait s'appeler la métalepse du commentateur ou encore - pour faire système avec mes propositions précédentes - métalepse métatextuelle; elle relève en tout cas de l'énonciation métatextuelle. Si je préfère la dénomination de métalepse du lecteur, c'est que l'instance du commentateur ne fait que dire la lecture qu'il fait du texte - on verra d'ailleurs que la métalepse est précisément comme une figure de la lecture - et que la transgression qui s'opère dans le métatexte n'est pas seulement un franchissement de la frontière entre l'énonciation textuelle et l'énonciation métatextuelle, mais entre cette dernière et l'énonciation narrative ${ }^{15}$.

Pour récapituler ce système terminologique :

métalepse du narrateur $=$ métalepse narrative (énonciation narrative)

métalepse de l'auteur $=$ métalepse textuelle (énonciation textuelle)

métalepse du lecteur $=$ métalepse métatextuelle (énonciation métatextuelle)

Si ce maillage un peu serré peut inquiéter, rappelons, avec Lavocat (2016) citée plus haut, qu'identifier des frontières ne veut pas nécessairement dire les fermer : cela peut même, au contraire, aider à mieux voir les effets de leur franchissement.

\section{La métalepse du lecteur}

Si Genette n'a pas exactement ignoré la métalepse du lecteur, il ne l'a pas identifiée spécifiquement - ce qui est normal pour un narratologue qui s'est donné pour projet de penser davantage le fonctionnement de la narration que sa réception. Cela conduit chez lui à une minoration du phénomène, qui peut se voir notamment dans la définition qu'il donne de la métalepse en 1983 (p. 58), déjà citée : elle se réalise, dit-il, « lorsqu'un auteur (ou un lecteur) s'introduit dans l'action fictive de son récit ou lorsqu'un personnage de cette fiction vient s'immiscer dans l'existence extradiégétique de 
l'auteur (ou du lecteur) ». La mise entre parenthèse (littérale) du lecteur signale un oubli de ce dernier dans la réflexion de Genette - au point que la syntaxe est bancale et que la parenthèse incluant le lecteur sonne comme un ajout: car pourrait-on dire qu' « un lecteur s'introduit dans l'action fictive de son récit ${ }^{16}$ »?

Mais s'il néglige d'isoler cette forme de métalepse, Genette en cite cependant des réalisations dans ses écrits, par exemple quand, à deux reprises (1969, p. 216 et 1972, p. 244), il donne le même exemple (de son cru) pour illustrer la métalepse de l'auteur (ou du narrateur), qu'il voit " lorsqu'on dit que Virgile "fait mourir" Didon au chant IV de l' Énéide»; ou encore quand il cite (1972, p. 244, note 2) cette citation de Boileau (Art poétique, I, 25-26) à propos de Saint-Amant: «Et poursuivant Moïse au travers des déserts / court avec Phaéton se noyer dans les mers ». Dans ces exemples, ce n'est pas l'auteur ou le narrateur qui transgresse une frontière, c'est le commentateur. Certes, on a déjà vu des exemples - et Genette (2004) en donne d'assez nombreux - où un lecteur fictif (fictivement présenté comme réel) s'immisce dans une fiction qu'il lit ${ }^{17}$ : mais on reste là dans la métalepse narrative, où un monde représenté représente à son tour un monde, avec des jeux de transgressions fictifs.

Ce dont il est question ici, sous le nom de métalepse du lecteur, relève bien d'une transgression qui s'effectue par le passage du réel du commentateur dans la fiction du monde représenté. Genette (2004) donne, comme en passant et par simple évocation, quelques rares exemples de métalepse du lecteur, par des incursions au théâtre : qu'on pense (cf. 2004, p. 52 sq.) aux spectateurs intervenant dans une pièce de théâtre, ce qui n'était pas incongru au XVII ${ }^{\mathrm{e}} \mathrm{s}^{18}$., ou au discours ordinaire d'un spectateur d'aujourd'hui " "accommodant" son attention tantôt sur la conduite du personnage, tantôt sur la performance du comédien », ce qui peut l'amener à dire : « Isolde entre en scène » (p. 57 sq. $)^{19}$.

Genette cite encore d'autres exemples de jeux fictifs de passages de frontières, comme dans le Capitaine Fracasse de Gautier, où le commentateur d'une pièce de théâtre passe insensiblement à l'analyse de l'état d'esprit des personnages de la pièce, comme si, spectateur « réel », il pouvait entrer dans la fiction elle-même - ce que rend visible le passage, dans le texte, du présent de commentaire au passé fictionnel (Genette, 2004, p. 41-47). Le choix des temps produit le même effet métaleptique dans Jean le bleu de Giono, quand le commentaire du tableau de Brueghel, La chute d'Icare, est fait au passé, comme si on passait "d'un mode descriptif (d'une scène dépeinte) au mode narratif (d'une scène vécue) » (Genette, 2004, p. 88). C'est une transgression du même ordre qui s'observe encore dans l'exemple que donne Genette (p. 47 sq.) d'un compte rendu journalistique utilisant le passé pour raconter l'intrigue, au lieu du présent de commentaire (le métatexte étant alors mis au même niveau temporel que l'acte de narration).

Dans ces derniers exemples, on reconnait des marqueurs de ce qui fait le propre de la fiction (Cohn, 1999/2001) : l'intrusion dans la conscience d'un personnage et l'usage de temps spécifiques, qui sont des signes du caractère non référentiel de la fiction (ibid.) On sait que Käte Hamburger (1957/1986) considère que le "prétérit épique »-auquel on peut identifier le passé simple français - est un marqueur de fictionnalité qui ne représente pas un temps et ne saurait dire le passé d'une fiction dont Hamburger postule l'atemporalité; en effet, il n'est pas référé à un "Je-origine » réel, comme c'est le cas pour tout énoncé de la réalité, mais à des « Je-origines » fictifs, le prétérit perdant alors toute signification de passé, contrairement au récit factuel (ibid., p. 82). Cette 
thèse fut reprise et élargie par Harald Weinrich (1964/1973), qui précisément identifie un double système des temps verbaux, sans rapport avec la temporalité mais avec une fonction différente : l'un (en particulier le passé simple) signale que le texte est fictif, l'autre (en particulier le présent) que le texte est un commentaire. C'est ce double système qui se voit mis à mal quand un commentaire utilise, pour emprunter ses termes à Weinrich (ibid, p. 44 et 39), les temps du «monde raconté » dans le «monde commenté » (comme, aussi, dans l'exemple de Bayard qui ouvrait cet article). Pour faire appel à un autre système théorique, Vuillaume (1990) - qui discute (p. 50-57) les thèses de Hamburger et de Weinrich - analyse comment le système temporel des récits rend compte du fait paradoxal que «tout se passe comme si le texte décrivait des évènements appartenant à une époque révolue et, simultanément, faisait du lecteur le contemporain de ces évènements » (ibid., p. 10) : l'effet métaleptique d'un commentaire au passé tient alors au fait que, usant du même système temporel que le récit fictif, le métatexte prétend, comme la fiction, "susciter chez le lecteur l'illusion que ces évènements se déroulent sous ses yeux " (ibid., p. 109) ${ }^{20}$.

Si donc Genette a identifié la métalepse du lecteur, il ne l'a pas isolée ni nommée et la traite généralement comme un phénomène de fiction, à l'exception de quelques rares exemples de métatextes non fictifs. Plus claire est l'identification du phénomène par Anaïs Goudmand (2016), qui parle de métalepse à propos du phénomène de collusion entre acteur et personnage de séries télévisées, qu'on peut voir se réaliser notamment quand un acteur doit quitter la série et qu'il s'agit "de trouver une motivation diégétique suffisamment forte pour le départ du personnage»(p.66): cela peut produire des effets métaleptiques dans les discours métatextuels des créateurs comme des spectateurs, par exemple quand ils mêlent les deux univers de la fiction et de la réalité dans un même énoncé ou quand ils désignent le personnage par le nom de l'acteur ou inversement.

31 Mais c'est, à ma connaissance, Sophie Rabau (2005) qui a le plus nettement décrit ce phénomène récemment, en commentant le titre d'un article de journal qu'elle cite de mémoire : "Bruno Peyron fait mieux que Jules Verne et Philéas Phogg»; pour Rabau, dans cet énoncé, « auteur, personnage et lecteur sont mis en parallèle et, en un espacetemps improbable, cette comparaison fait sens » (p. 59). Ce que décrit Rabau ici est bien une métalepse métatextuelle, une métalepse du lecteur ${ }^{21}$, dont elle donne d'autres exemples, dont celui qui explique le titre de son article, et que l'on trouve chez Blanchot : «Quand Ulysse devient Homère » (p. 62).

Rabau cependant, comme on l'a vu plus haut avec Genette, me semble elle-même réaliser une figure du même ordre quand elle identifie, dans son article, les métalepses du lecteur réalisées dans un métatexte réel (qu'elle traite rapidement) de celles qui se jouent dans des œuvres de fiction, comme quand, dans L'Histoire véritable de Lucien, Ulysse côtoie Homère et Lucien; ou quand Homère s'entretient avec Achille dans le Dialogue des morts de Fénelon. Certes, Rabau distingue les cas de métalepses hypertextuelles (comme ces derniers exemples) et ceux de métalepses métatextuelles (comme les premiers exemples donnés), mais l'assimilation qu'elle opère entre les deux me semble elle-même métaleptique ${ }^{22}$...

Il me semble au contraire nécessaire de distinguer les métalepses du lecteur réalisées dans un contexte fictif des métalepses qui se réalisent dans le contexte d'une écriture métatextuelle réelle: dans le premier cas, c'est une forme spécifique de ce jeu métaleptique autorisé voire induit par la fiction; de l'autre, c'est une irruption du 
fictionnel dans le factuel - qui par ailleurs peut avoir, comme toute métalepse, un caractère rhétorique ou ontologique ${ }^{23}$. Si la métalepse du lecteur, comme toute métalepse, « tantôt accentue, tantôt arase, mais toujours fictionnellement, la différence entre les mondes » (Lavocat, 2016, p. 471), ce n'est pas la même chose de le faire au sein ou hors d'une fiction.

\section{Discours métaleptiques}

Tous les exemples donnés en illustration de la métalepse du lecteur ${ }^{24}$ montrent l'extrême banalité du phénomène et sa consubstantialité avec l'écriture métatextuelle sur un texte de fiction ${ }^{25}$. Il me semble qu'il y a là deux raisons : la première est que la métalepse du lecteur est assez naturellement liée à tout acte de lecture, qui mêle, le temps de son appréhension, le monde du texte au monde du lecteur; la deuxième est liée à la nature même de l'énonciation métatextuelle, qui se doit d'incorporer dans son propre tissu des éléments du texte commenté. Ce sont ces deux raisons que je me propose d'examiner rapidement.

\section{Métalepse et lecture}

Genette (1969, p. 216), reprenant l'un des sens de l'étymon grec de métalepse, écrit que «la métalepse de l'auteur ou du narrateur est proprement une figure de participation»: elle consiste, finalement, en «la participation du narrateur à son récit ». La métalepse du lecteur est bien cette participation du commentateur au récit et ne fait que rendre compte de la "participation du lecteur » au texte dont parle Bayard (1998/2002), «le texte se constituant pour une part non négligeable des réactions individuelles de tous ceux qui le rencontrent et l'animent de leur présence " (ibid., p. 130). De son côté, Dufays $\left(1994 / 2010\right.$, p. 176) appelle participation ${ }^{26}$ "la lecture qui cherche avant tout à saisir ce qui dans le texte est représentable »; il ajoute : "Appliqué à un récit, ce mode de lecture se focalise avant tout sur le "suspense", sur le caractère "humain" des personnages et les actions concrètes qu'ils effectuent ${ }^{27}$."

La métalepse du lecteur peut être comprise comme l'expression de cette participation, que suppose l'appropriation - ou l'application - propre à toute lecture, dans une approche herméneutique que reprend Ricœur $\left(1985^{28}\right)$; pour ne faire qu'évoquer son parcours des trois mimesis (1983, p. 107), rappelons que Ricœur, au-delà de la «configuration textuelle » (qu'il nomme mimesis II), envisage l'amont de l'œuvre (mimesis I, entendue comme " préfiguration du champ pratique ») et son aval (mimesis III, « refiguration par la réception de l'œuvre »). C'est ainsi que « mimesis III marque l'intersection du monde du texte et du monde de l'auditeur ou du lecteur, l'intersection donc entre monde configuré par le poème et monde au sein duquel l'action effective se déploie » (1983, p. $136 ; 1985$, p. 287). On pourrait dire, pour trivialiser l'usage d'un terme herméneutique (voir Ricœur, 1985, p. 399) que la fusion énonciative que réalise la métalepse du lecteur rend compte de la fusion d'horizons en quoi consiste la lecture ou le discours sur la lecture ${ }^{29}$.

La métalepse du lecteur constituerait une manifestation de ce trajet herméneutique. Pour reprendre les termes de Sophie Rabau (2005, p. 68), la figure qu'elle appelle hétérométalepse et que je nomme métalepse du lecteur «répond au propos de l'interprétation en ce qu'elle abolit la distance entre, d'une part, l'intériorité et 
l'extériorité de l'œuvre et, d'autre part, entre l'espace-temps de l'interprétation, l'espace-temps de la fiction et celui de l'œuvre fictive ». Rabau (2005), on l'a vu plus haut, décrit un phénomène plus large, mais je m'empare de ses analyses pour caractériser les relations entre la lecture et la métalepse du lecteur dans un contexte métatextuel réel ${ }^{30}$. En effet, si cette figure « réunit [...] en un seul univers le monde de la production, de la fiction et de la réception ", elle se rapproche de l'interprétation, voire de "la lecture la plus élémentaire ", qui "peut se décrire comme une mise en rapport de ces trois univers» (ibid, p. 60). Rabau ajoute (ibid, p. 60 sq.) : «Elle a pour propos de donner sens ici et maintenant au texte, écrit autrefois et ailleurs, où l'on narre une histoire qui a lieu encore en un autre temps et en un autre lieu.» C'est pourquoi le phénomène, pour étrange qu'il soit quand on l'analyse (ibid, p. 61),

serait moins une exception, une figure qui s'écarte de l'usage, qu'une manière d'accomplir ce dessein herméneutique presque banal. Représenter un monde où se côtoient et dialoguent l'auteur, les personnages et le lecteur, reviendrait, en d'autres termes, à se donner une manière d'utopie qui pousserait à l'extrême la démarche de toute interprétation du texte littéraire.

La dimension métaleptique de la lecture, qui pourrait se légitimer des nombreuses théories de la lecture ou de la fiction qui mettent en avant l'interaction du texte et du lecteur dans la construction du sens des textes, impliquerait, pour que le processus de compréhension soit possible, une sorte de suspension de réalité - garante de la suspension consentie de l'incrédulité de Coleridge, si souvent cité.

Yves Citton (2010) a tiré un parti particulier de cette dimension métaleptique de toute lecture, quand il avance le concept de scénarisation pour décrire (p. 84) l'agencement, dans un scénario ${ }^{31}$, des actions de personnages fictifs comme des actions de personnes réelles (p. 85):

Une activité de scénarisation peut donc s'appliquer à la fois à des personnages fictifs joués par des acteurs et à mes propres comportements d'individu réel.

Or, dit Citton, « la scénarisation repose sur ce que la narratologie appelle une métalepse » ibid.) ; ou, en d'autres termes : « L'activité de scénarisation est métaleptique » (ibid, p. 86). La transgression métaleptique s'opère en fait de deux manières; d'une part, dans le sens du lecteur vers le texte (p. 87):

Lire un roman, écouter un conte, regarder un film, cela implique de projeter ses explications causales, ses affects et ses valorisations (éthiques, politiques) dans les enchainements d'évènements dépeints par le récit.

D'autre part, dans le sens du texte vers le lecteur, dans la mesure où « les récits agissent sur ceux qui les écoutent» (p. 84); c'est ainsi que s'en explique Citton (p. 87), dont l'ouvrage, rappelons-le pour bien comprendre les mots qui suivent, porte sur le storytelling:

Toute histoire qui « passe » est orientée par un faire-faire (faire-rire, faire-pleurer, faire peut, faire-dire, faire-acheter, faire-s'indigner, faire-s'engager, faire-voter). Un récit, en ce sens, fonctionne bien comme un prompteur : un stimulateur d'action, un déclencheur et un conducteur de conduite. L'enchainement d'actions (fictives) représenté par le récit vise donc à produire un certain enchainement d'actions dans la réalité à venir.

On aura observé que Citton ignore la frontière entre réalité et fiction de deux façons, d'une part en ne distinguant pas, dans la description du processus de scénarisation, les récits selon qu'ils sont fictifs ou non, d'autre part en traitant de la même manière l'action dans le monde fictif et dans le monde réel. 
Cette position s'explique par le projet de Citton, dont l'ouvrage porte sur le storytelling, dans une filiation avec de nombreux auteurs sur la question qui, au rebours de la plupart des narratologues, «tiennent généralement pour acquise l'indifférenciation entre fait et fiction, dans une optique panfictionnaliste qui dissout les frontières de la fiction et la notion de fiction elle-même ", pour citer Lavocat (2016, p. 33) qui présente cette position pour la réfuter.

Mais on peut au contraire considérer que la dimension métaleptique de la lecture fait ressortir cette frontière entre fictionnel et (f)actuel, pour emprunter le quasi-motvalise de Jean-Marie Schaeffer $(2005$, p. 325), qui interroge précisément la caractéristique de l'immersion fictionnelle, qu'il considère comme "un état mental scindé » (p. 325 ; 333), un "état clivé » (p. 333) : «dans l'immersion fictionnelle, le monde fictif ne prend pas la place du monde réel: il y a coexistence entre les deux mondes» (p 332). Et si «la dynamique de l'immersion implique des opérations mentales métaleptiques» (p. 333), c'est que toute lecture peut reconnaitre, en la transgressant, la frontière du fictionnel et du réel.

5 Quelle que soit la position que l'on prend sur cette question, il me semble en tout cas possible d'identifier la métalepse du lecteur dans un discours métatextuel comme la trace de la métalepse de la lecture.

\section{Métalepse et greffe métatextuelle}

4 La métalepse du lecteur est un phénomène énonciatif qui met en jeu des frontières énonciatives dans le cadre d'un contexte fictif, en incorporant du fictif dans le métatexte. Il faut observer que ce jeu des frontières énonciatives n'est pas sans lien avec celui qui s'observe dans tout discours qui incorpore celui d'autrui au point de fondre l'un dans l'autre, pour ainsi dire: c'est particulièrement le cas du discours indirect libre, qui réalise à sa manière, dans un énoncé fictif ou non, littéraire ou non, la fusion des énonciations dans le discours, quand un énonciateur prend en charge l'énonciation d'un autre. Mais c'est encore le cas de tout discours métatextuel voire de tout énoncé métalinguistique, où peut se réaliser un franchissement des frontières énonciatives, entre le métalangage et le langage qu'il prend pour objet ${ }^{32}$.

47 Un tel franchissement peut s'avérer parfois problématique: c'est le cas quand il se réalise dans le métalangage scientifique, comme l'a montré Oswald Ducrot (1984), qui a analysé ce phénomène pour " dénoncer la confusion [...] entre le langage qu'on étudie et le métalangage au moyen duquel on l'étudie» qu'il observe en linguistique sémantique et particulièrement en pragmatique. Cette confusion tient, dit Ducrot, « à la nécessité, dans ces domaines de recherche peu formalisés, d'utiliser, pour décrire une langue, les mêmes mots et les mêmes structures syntaxiques appartenant à la langue qui est l'objet de l'investigation » (1984, p. 117 sq.). Mais « c'est oublier que ces mots, employés dans un discours théorique, reçoivent de ce fait un statut de concepts théoriques qu'ils n'avaient pas dans l'usage quotidien » (ibid., p. 118).

Ducrot illustre le problème par un exemple (ibid., p. 120) : imaginons un locuteur (L) dire, à propos d'une personne $(\mathrm{P}):$ «P est intelligent ». Si le linguiste rend compte de cet évènement en usant du discours direct « $\mathrm{L}$ a dit : "P est intelligent." ", il n'y a pas de souci ; c'est autre chose si le linguiste écrit, en utilisant le discours indirect : « L a dit que P est intelligent. » Car, dans ce cas, le linguiste « doit utiliser le mot intelligent à ses propres frais », autrement dit « le revendiquer comme un concept scientifique, pourvu 
d'une valeur théorique claire, et désignant effectivement soit une propriété, soit un ensemble » (ibid., p. 121). Sans doute n'était-ce pas son intention, mais c'est bien ce qui se produit quand il use d'une telle manière de parler ordinaire.

Ce faisant, le linguiste a alors reproduit une dérivation propre au langage, qui a une propension à la «fabrication d'une propriété à partir d'un discours". En effet, pour Ducrot (ibid., p. 123), ce n'est que par dérivation que l'énoncé, purement argumentatif au départ, peut - et doit dans la langue d'usage - être réinterprété comme une affirmation, désignant $\mathrm{P}$ comme possédant une propriété, celle qui justifie l'argumentation dont l'énoncé est porteur. Cette dérivation, Ducrot l'appelle dérivation délocutive ou délocutivité33.

C'est à partir de cette dérivation délocutive ou délocutivité élargie de Ducrot que j'avais, au départ $(1993 ; 2002 b)$, forgé les termes de dérivation détextuelle ou détextualité pour désigner le phénomène par lequel un discours sur un texte intègre un effet du texte : c'est le cas lorsqu'un métatexte intègre un élément de la fiction, lui donnant comme le statut d'une réalité. Si j'opte aujourd'hui pour l'expression métalepse du lecteur, c'est d'abord en raison de l'extension qu'a prise le concept de métalepse dans la littérature théorique; mais c'est surtout pour éviter une ambigüité que contenait la notion de détextualité, sur laquelle je me propose de revenir ${ }^{34}$.

L'ambigüité tient au fait que cette notion, telle que je l'avais construite, mélangeait les modalités et les effets de la (con)fusion énonciative à l'œuvre dans le métatexte. Les modalités concernent la manière dont est effectuée la greffe ${ }^{35} \mathrm{du}$ texte dans le métatexte, selon que l'énonciation métatextuelle se fond dans celle du texte-source (c'était le cas de la paraphrase stricto sensu, telle que je la décrivais alors) ou que l'énonciation du texte-source s'efface au profit de celle du métatexte (c'était la détextualité). Les effets de la détextualité étaient l'intégration d'une caractéristique textuelle, à savoir le fait que la fiction semble réelle, dans le métatexte, effets que je décris dans le présent article sous l'expression de métalepse du lecteur.

Or celle-ci peut également être réalisée dans le cas d'une paraphrase! De même, la modalité énonciative inverse (quand le discours métatextuel régit le discours cité) n'implique pas nécessairement une métalepse du lecteur. Autrement dit, contrairement à ce que j'avançais dans mes premières descriptions du phénomène, il convient de dissocier modalités énonciative et effet métaleptique : quelles que soient les modalités d'intégration du discours d'autrui dans un métatexte (effacement de l'énonciation du discours cité ou du discours citant), l'effet métaleptique peut ou non se réaliser.

53 Pour éviter la projection que je faisais de la détextualité (modalité énonciative) sur la métalepse du lecteur (effet), je préfère désormais, suite à un travail mené avec Isabelle Delcambre (Daunay et Delcambre, 2016 ; à paraitre), clarifier cette terminologie. Sur le plan des modalités énonciatives de la reprise dans un texte du discours d'autrui, nous avons proposé d'identifier deux pôles, entre lesquels peuvent varier les degrés de prise en charge énonciative du discours d'autrui dans un métatexte: la métaphrase et la paraphrase; dans la métaphrase, c'est le discours métatextuel qui prend en charge totalement le propos d'autrui, dont il régit l'énonciation; dans la paraphrase, c'est l'énonciation du texte-source qui régit celle du métatexte.

Nous avons construit ces deux pôles pour le discours métatextuel académique, mais la description vaut pour n'importe quel genre de discours qui prend celui d'un autre pour objet et, bien évidemment, pour le commentaire littéraire, où se joue notre métalepse du lecteur. Prenons, pour illustrer ce dernier contexte, des exemples très contrastés 
empruntés à un corpus que j'ai analysé ailleurs (Daunay, 2017), constitué d'énoncés métatextuels du PISA à propos de textes littéraires ${ }^{36}$.

Dans l'encadré qui suit, je place ces exemples à des degrés divers entre les deux pôles, de manière toute intuitive :

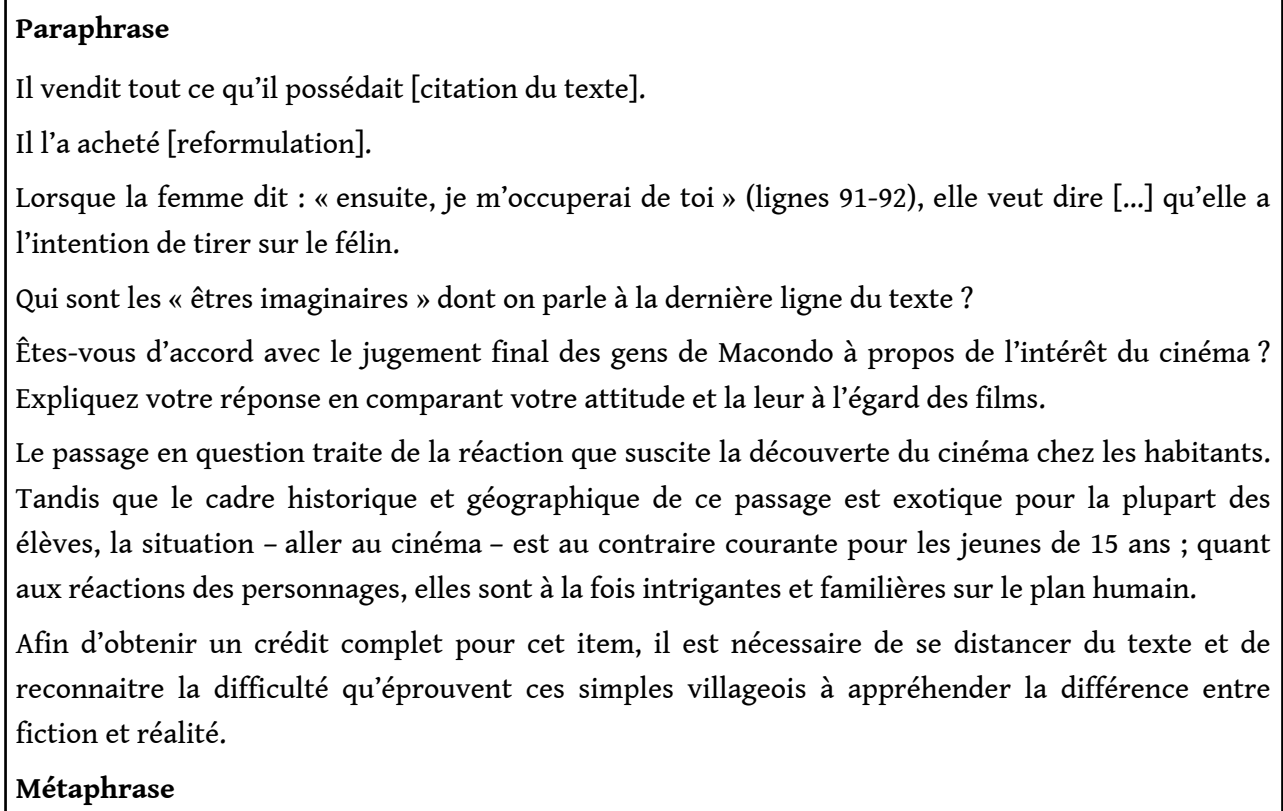

Dans ce système, la citation et la reformulation non marquées (au point qu'il a fallu que je signale leurs natures entre crochets) sont les cas où le métatexte délègue le plus la prise en charge énonciative au texte source, puisque c'est l'énonciation du texte source qui, tout entière (dans le cas de la citation) ou presque entièrement (pour la reformulation), régit l'énonciation du métatexte, jusque dans les temps des verbes. Inversement, dans les derniers exemples, les éléments du texte-source sont comme fondus dans le métatexte, qui reprend totalement à son compte les objets du texte (aucun terme exact du texte-source n'est repris, même si l'on reconnait ce dont il y est question) dans un discours au présent.

57 Ces modalités énonciatives contrastées, qui vont de la plus pure paraphrase à la plus pure métaphrase, avec toutes les nuances entre les deux, produisent pourtant le même effet métaleptique. De fait, dans tous les cas, quelle que soit la modalité, on observe un effet de confusion entre monde du texte et monde du métatexte, entre récit fictif et commentaire réel. Et c'est cette confusion-là qui est une métalepse du lecteur.

Peut-être faudrait-il encore préciser les choses, en reprenant la terminologie utilisée supra: finalement, les jeux de frontière entre l'énonciation textuelle et l'énonciation métatextuelle, qui concernent les modalités, n'ont un effet métaleptique que quand elles engagent un autre jeu de frontière : entre l'énonciation métatextuelle et l'énonciation narrative, où se joue la fiction. 


\section{Conclusion}

Il est d'un usage bien ancré désormais d'affirmer la porosité du métatexte pour la réputer propre à la postmodernité. Mais ce qui est propre aux auteurs qui s'en réclament n'est pas tant la "contamination entre texte et métatexte postmodernes " (Lamontagne, 1998, p. 63) que la revendication de cette contamination. Elle va de pair avec la revendication d'une "contamination de la théorie par la fiction", selon les termes de Lavocat (2016, p. 522), qui la dénonce comme curieuse.

Mais si la revendication peut être considérée comme nouvelle (quand du moins l'on s'en tient au temps court historique), la réalisation effective de ces contaminations est consubstantielle au discours métatextuel. Comme le dit Dion (1998, p. 82) à propos de la critique savante, y compris structurale : «Quel article, tout bien pesé n'élabore pas un métadiscours plus ou moins narrativisé, ni ne relate l'œuvre lue?» Quand cette contamination engendre, dans le métatexte, une confusion entre le monde fictif du texte et le monde réel du lecteur identique à celle qui s'opère dans toute lecture, on peut parler de métalepse du lecteur.

Je reprendrai pour finir les mots de Frank Wagner qui, parlant de métalepse narrative (2002, p. 250 sq.), me semble exprimer assez bien ce que l'on pourrait dire de la métalepse du lecteur :

La prise en compte des ambigüités générées par certains usages de la métalepse permet de rappeler que le monde et la fiction ne sont pas des univers rigoureusement forclos, mais qu'ils se trouvent engagés dans un procès d'échanges réciproque.

62 C'est, je crois, ce "procès d'échanges réciproque » que constitue la métalepse du lecteur, par lequel ce dernier, devenu commentateur, se fait commenteur, celui qui ment avec l'auteur ${ }^{37}$.

\section{BIBLIOGRAPHY}

Authier-Revuz, J. (1998/2013). Ces mots qui ne vont pas de soi. Boucles réflexives et non-coïncidences du dire. Limoges : Lambert-Lucas.

Baroni, R. (2007). La tension narrative. Suspense, curiosité et surprise. Paris : Seuil.

Bayard, P. (1998/2002). Qui a tué Roger Ackroyd ?. Paris : Minuit.

Benveniste, É. (1959/1966). Les verbes délocutifs. In Problèmes de linguistique générale, tome 1. Paris : Gallimard, p. 277-295.

Blumenfeld, R. (1980). Remarques sur Songe / Mensonge. Romania, 101/403, 385-390.

Charles, M. (1978). La lecture critique. Poétique, 34, 129-151.

Citton, Y. (2010). Mythocratie. Storytelling et imaginaire de gauche. Paris : éditions Amsterdam.

Cohn, D. (1999/2001). Le propre de la fiction. Paris : Seuil. 
Daunay, B. (1993). Les questions de compréhension au brevet des collèges. Fonctionnement et fonction d'un type spécifique de métatexte. Recherches, 19, 101-120.

Daunay, B. (2002a). Éloge de la paraphrase. Saint-Denis : Presses Universitaire de Vincennes.

Daunay, B. (2002b). La Paraphrase dans l'enseignement du français. Neuchâtel : Peter Lang.

Daunay, B. (2002c). Le lecteur distant. Positions du scripteur dans l'écriture du commentaire. Pratiques, 113-114, 135-153.

Daunay, B. (2017). La métalepse du lecteur dans le métatexte du PISA. forumlecture.ch, 2/2017.

Daunay, B., Delcambre, I. (2016). Les modalités énonciatives de la reformulation. Comparaison entre écriture d'enseignement et de recherche. Langues, cultures et sociétés, 2/1, en ligne : http:// revues.imist.ma

Daunay, B., Delcambre, I. (à paraitre). Les modalités énonciatives de la reprise du discours d'autrui dans les écrits de recherche et les écrits didactiques. Scripta, 21/43.

Dion, R. (1998). La narrativité critique. Études littéraires, 30/3, 77-90.

Ducrot, O. (1984). Le dire et le dit. Paris : Minuit.

Dufays, J.-L. (1994/2010). Stéréotype et lecture. Essai sur la réception littéraire, Bruxelles, Peter Lang. Dumarsais, C. C. (1730/1818). Traité des tropes. Paris : Belin-le-Prieur.

Fitch, B. (2000) À l'ombre de la littérature, Montréal, XYZ éditions.

Fontanier, P. (1821/1968). Les Figures du discours. Paris : Flammarion.

Franko, M. (1993/2005). La danse comme texte. Idéologies du corps baroque. Paris : Kargo \& L'Éclat.

Genette, G. (1969) Figures II. Paris : Seuil.

Genette, G. (1972). Figures III. Paris : Seuil.

Genette, G. (1983). Nouveau discours du récit. Paris : Seuil.

Genette, G. (1991). Fiction et diction. Paris : Seuil.

Genette, G. (2004). Métalepse. De la figure à la fiction. Paris : Seuil.

Goudmand A. (2016). « Oh my God! They’ve killed ! " Le récit sériel entre autonomie et hétéronomie : conséquences du départ non planifié des acteurs. Télévision, 7, 65-83.

Hamburger, K. (1957/1986). Logique des genres littéraires. Paris : Seuil.

Lafarge, C. (1983). La valeur littéraire. Figuration littéraire et usages sociaux des fictions. Paris : Fayard. Lamontagne, A. (1998). Métatextualité postmoderne : de la fiction à la critique. Études littéraires, $30 / 3,61-76$.

Lavocat, F. (2016). Fait et fiction. Pour une frontière. Paris : Seuil.

Nelles, W. (1997). Frameworks. Narrative Levels \& Embedded Narrative, New York, Peter Lang.

Patron, S. (2009/2016) Le Narrateur, un problème de théorie narrative. Paris : Lambert-Lucas.

Perelman, C., Olbrechts-Tyteca, L. (1958). La nouvelle rhétorique. Traité de l'argumentation. Paris :

Presses universitaires de France.

Pier, J. (2005). Métalepse et hiérarchies narratives. In J. Pier, J.-M. Schaeffer (eds) Métalepses.

Entorses au pacte de la représentation, Paris, EHESS (p. 247-261). 
Rabau, S. (2005). Ulysse à côté d'Homère. Interprétation et transgression des frontières énonciatives. In J. Pier, J.-M. Schaeffer (eds) Métalepses. Entorses au pacte de la représentation, Paris, EHESS (p. 59-72).

Rey-Debove, J. (1978/1997) Le métalangage. Étude linguistique du discours sur le langage, Paris, Armand Colin.

Ricœur, P. (1983). Temps et récit. 1. L'intrigue et le récit historique. Paris : Seuil.

Ricœur, P. (1985). Temps et récit. 3. Le temps raconté. Paris : Seuil.

Rigolot, F. (1990). Introduction à l'étude du « commentataire ». L'exemple de la Renaissance. In G. Mathieu-Castellani, M. Plaisance (éd.). Les commentaires et la naissance de la critique (p. 51-62). Paris : Aux amateurs du livre.

Ryan, M.-L. (2005). Logique culturelle de la métalepse, ou la métalepse dans tous ses états. In J. Pier, J.-M. Schaeffer (eds) Métalepses. Entorses au pacte de la représentation, Paris : EHESS (p. 201-223).

Ryan, M.-L. (2010). Cosmologie du récit : des mondes possibles aux univers parallèles. In F. Lavocat (ed) La théorie littéraire des mondes possibles, Paris : CNRS éditions (p. 53-81).

Schaeffer, J.-M. (2005). Métalepse et immersion fictionnelle. In J. Pier, J.-M. Schaeffer (eds) Métalepses. Entorses au pacte de la représentation, Paris, EHESS (p. 323-334).

Vuillaume, M. (1990). Grammaire temporelle du récit. Paris : Minuit.

Wagner, F. (2002). Glissements et déphasages : Note sur la métalepse narrative. Poétique, 130, 235-253.

Weinrich, H. (1964/1973). Le Temps. Paris : Seuil.

\section{NOTES}

1. Que j'avais ouvert, de mon côté, dans un article de 1993 sur les questions de compréhension à l'examen terminal du secondaire inférieur en France (le «brevet des collèges »). Cet article de 1993 a fait l'objet de reprises dans deux ouvrages parus une dizaine d'années plus tard (2002a ; 2002b). Je parlais alors de détextualité - et je m'expliquerai sur ce terme plus loin, de même que je justifierai ma proposition de parler désormais plutôt de métalepse du lecteur.

2. Je le fais, en analysant un corpus spécifique, celui du PISA, dans un récent article: Daunay (2017).

3. Ce texte applique les rectifications orthographiques du français proposées en 1990 par le Conseil supérieur français de la langue française. Merci à Carole-Anne Deschoux pour son accompagnement dans la réalisation de cet article.

4. Il emploie l'adjectif hardi en référence ludique à l'usage du mot par Fontanier, qu'on rencontrera bientôt.

5. Cette distinction de Ryan ne recoupe pas exactement celle que proposait Nelles en 1997 (p. 155), entre métalepse épistémologique (ou verbale) et métalepse ontologique (ou modale), selon que les personnages passent effectivement d'un niveau narratif à l'autre ou que ce dernier est simplement évoqué, par exemple par la connaissance que les personnages ont des évènements survenus dans un autre univers diégétique que le leur. Je traduis les termes de Nelles: «Ontological (or modal) and epistemological (or verbal)» (noter que, p. 134, Nelles proposait plutôt epistemic, ce qui me semble plus pertinent). Dans le système de Nelles, cette opposition ne concerne pas la «métalepse rhétorique», qui n'est qu'une «basic "unmarked" case of 
metalepsis» (p. 153). Pour d'autres approches typologiques, voir Wagner (2002), Pier (2005, p. 249-252) et surtout Lavocat (2016, 473-520).

6. Lui-même inspiré de César Chesneau Dumarsais (1730/1818, p. 111) : « On raporte aussi à cette figure ces façons de parler des Poètes, par lesquelles ils prènent l'antécédent pour le conséquent, lorsqu'au lieu d'une description, ils nous mètent devant les yeux le fait que la description supose ». Je respecte à dessein l'orthographe de Dumarsais - et je crois bien même que c'est pour cela que je tiens à faire cette citation... -, car elle résulte chez lui d'un choix d'illustration d'une "nouvèle orthographe ", qu'il appelait de ses vœux (cf. les remarques qu'il fait à ce sujet, en ouverture de son ouvrage, sous le titre « Errata », p. 18-20).

7. La dénomination métalepse de l'auteur semble bien de Genette. En 1972 (p. 244), il l'attribue aux " classiques", sans plus de spécifications, mais il reconnaitra plus tard (2004, p. 14) que cette attribution était indue, même s'il considère « cette expression fidèle, sur le fond, aux analyses de la rhétorique classique ».

8. Pour prendre l'exemple de Manon Lescaut que Genette suggère pour décrire (1972, p. 238 sq.), l'extradiégétique, niveau narratif qui fait système avec le niveau diégétique (ou intradiégétique) et les niveaux métadiégétiques : le niveau extradiégétique est constitué par l'acte littéraire fictif que constitue la rédaction des Mémoires de Renoncour; Prévost, lui, se situe ailleurs que dans l'extradiégétique, puisqu'il ne relève pas de la fiction. Mais il est vrai que, à ce même endroit, Genette réalise une métalepse, puisqu'il suppose que Renoncour «s'adresse, quoique fictif, au public réel »...

9. Pour détourner l'expression de Perelman et Olbrechts-Tyteca (1958).

10. Je reprends, en le modifiant sensiblement, la figure que je propose dans Daunay (2002b, p. 159).

11. Qui peut être contestée, notamment par le fait qu'elle induise benoitement une conception communicationnelle du récit, souvent discutée et encore récemment, assez magistralement, par Sylvie Patron (2009/2016) ; mais, de fait, on s'en est sans doute déjà aperçu, c'est bien dans un tel paradigme que je me situe pour mieux (ou plus facilement) identifier les jeux de transgression de la frontière entre fictionnel et réel: c'est ce qui explique que, comme le fait la narratologie traditionnelle, j'assigne bien à tout récit une voix qui assume la narration, soit un narrateur relevant de la fiction si le récit est fictif.

12. Ce que j'appelle ici énonciation narrative, pour faire système avec les autres termes, est ce que Genette considère comme une énonciation au niveau extradiégétique.

13. Sauf erreur, le mot, calqué sur une série déjà cohérente (destinataire, narrataire) est de Rigolot (1990, p. 52).

14. Chaque acte de parole des personnages au niveau intradiégétique peut ainsi être entendu comme un récit (sur ce point et plus généralement sur la question des récits enchâssés, cf. Nelles, 1997). Ryan propose un autre modèle de représentation ces jeux de frontières, la pile ou "stack », qu'elle dit emprunter à la science informatique (2005, p. 205), modèle qui facilite la représentation des enchâssements successifs.

15. La confusion qui fait que le métatexte se confond avec le texte et que le commentateur fait comme s'il en était l'auteur relève des jeux énonciatifs liés à la reprise du discours d'autrui dans son texte : cela n'est pas étranger à la métalepse mais ne pose pas les mêmes questions que celle que pose la métalepse du lecteur en contexte fictionnel. Voir ci-dessous, 2.2.

16. C'est moi qui souligne.

17. Quand Genette parle du lecteur, c'est en fait du narrataire : s'il écrit en effet que «cette capacité d'intrusion dans la diégèse, dont l'auteur use à sa guise, peut aussi bien s'étendre à cet autre habitant de l'univers extradiégétique qu'est le lecteur ", c'est en fait pour parler de "l'introduction (évidemment fictive) du lecteur potentiel extradiégétique dans la diégèse fictionnelle » (2004., p. 95). C'est à l'occasion de ce jeu fictionnel, qu'illustre notamment l'adresse 
en "vous" au lecteur, que Genette use, pour la seule fois à ma connaissance, de l'expression « métalepse du lecteur » (ibid., p. 99), dans un sens différent de celui que je donne à l'expression. 18. Ou dans le ballet classique : voir à ce sujet ce que dit Mark Franko (1993/2005) des effets à la fois esthétiques et politiques de l'intégration du roi-spectateur, où une telle métalepse fait que « le spectacle cesse de représenter quelque chose à interpréter pour devenir l'interprétation en acte de la réalité historique » (p. 58).

19. Le cas du spectateur intervenant dans une pièce est évoqué encore par Genette (2004, p. 59), avec une allusion au soldat de Baltimore chez Stendhal (évoqué dans Racine et Shakespeare) ou à Don Quichotte (qui brise les marionnettes de Maitre Pierre dans le chapitre 26 du tome 2). Lavocat a bien montré que l'extension de la métalepse narrative pose quelques problèmes théoriques; cela apparait notamment dans le cas du théâtre, où le franchissement de la frontière entre la salle et la scène n'est pas du même ordre que celui qui s'opère entre les niveaux narratifs: "Alors que, pour tous les autres médias, l'obstacle de la page ou de l'écran est infranchissable, il n'y en a aucun entre la scène et la salle, ni matériel, ni ontologique » (2016, p. 487).

20. Ces marqueurs internes de fonctionnalité ont pu être contestés, y compris par des auteurs qui maintiennent la différence entre faits et fictions au profit de marqueurs externes, comme la distinction entre auteur et narrateur et la dimension figurale non sérieuse de l'énoncé (cf. Genette, 1991), mais il me semble précisément que l'usage de ces marques hors de la fiction produit un effet d'étrangeté (métaleptique) qui donne un surcroit de pertinence à la théorie...

21. Qu'elle nomme, de son côté, hétérométalepse.

22. Noter que Wagner (2002), quand il parle de la métalepse « comme une variété particulière de métatextuel » (p. 250), traite en fait de la dimension hypertextuelle, s'agissant de métatextualité dans l'œuvre : cette dimension métatextuelle et métaleptique reste en effet régie par l'auteur.

23. Par exemple, «Virgile fait mourir Didon au chant IV de l'Énéide» vs «Qui a tué Roger Ackroyd?» (le temps des verbes étant un indice assez sûr du partage de ces métalepses).

24. On en trouvera d'autres réalisations dans un corpus analysé de façon systématique dans Daunay (2017).

25. Je renverrai, pour illustrer cette idée, à mes descriptions de métatextes d'auteurs canoniques, d'époques différentes, comme Paul Bénichou ou Roland Barthes (Daunay, 2002a), d'amateurs éclairés, comme André Miquel (Daunay, 2002c) ou Paul Veyne (Daunay, 2002b), d'une didacticienne, Annie Rouxel (Daunay, 2002c), de parascolaires (Daunay, 2002c) ou de sujets d'examen (Daunay, 1993 ; 2002b).

26. Dufays dit emprunter, dans cette acception, le terme de participation à Lafarge (1983).

27. Ce qui est en jeu ici est «l'engagement affectif» des lecteurs "vis-à-vis des productions sémiotiques ou discursives» (Baroni, p. 22), qui est comme le garant de la "tension narrative » que suscite la mise en intrigue. En résulte ce « recentrement » que décrit Marie-Laure Ryan, par lequel «le lecteur (ou spectateur) se transporte en imagination dans le monde fictionnel et s'imagine appartenir à ce monde » (2010, p. 58). Pour rendre justice à Ryan et à quelques autres auteurs qui ont été mis à contribution pour la rédaction de cet article, il faudrait mettre en relation la métalepse du lecteur et les théories des mondes possibles. Mais cela dépasserait les limites de ce papier comme des compétences son auteur...

28. C'est Ricœur qui associe les termes d'application et d'appropriation (1985, p. 286).

29. Sur ce point, voir Fitch (2000, p. 331 sq.).

30. Qui, disais-je plus haut, me semble devoir être distinguée des autres cas (fictifs) qu'elle décrit.

31. Que le scénario soit narratif (dans le cas d'un récit) ou prospectif (comme quand on envisage un scénario de crise, par exemple).

32. Qu'on se place dans les perspectives (proches quoique différentes) d'Authier-Revuz (1995/2013) ou de Rey-Debove (1978/1997). 
33. Il emprunte l'expression à Benveniste (1958/1966), qui décrivait la dérivation d'un terme à partir d'une expression du discours, comme « remercier » à partir de « merci ! »

34. Je remercie Daniel Bart d'avoir mis le doigt sur cette ambigüité, dans nos échanges sur le métatexte du PISA et sur l'écriture théorique. Merci encore à lui pour sa lecture attentive des différents états du présent article.

35. La métaphore de la greffe se trouve chez Charles (1978), souvent reprise ensuite. Je ne peux que renvoyer ici, sans pouvoir développer, aux intéressantes remarques de Fitch (2000) sur le métatexte (cf. notamment p. 311 sqq.).

36. Le PISA est le Programme international de suivi des acquis des élèves (traduction approximative de l'anglais Programme for International Student Assessment). Je ne donne pas ici les références exactes des énoncés que je lui emprunte, mais, si besoin, on les trouvera facilement en les recopiant dans un moteur de recherche sur l'Internet (ou en se reportant à mon article cité : Daunay, 2017).

37. Aucune valence morale évidemment, dans le choix de ce terme : simplement un jeu de mots qui renvoie à un autre jeu de mots reliant (sémantiquement et non étymologiquement), dans les proverbes comme dans la littérature, mensonge à songe (Blumenfeld, 1980), termes qui peuvent tous deux renvoyer à une illusion (cf. Trésor de la langue française, s.v., à qui j'emprunte l'idée et la référence de cette note).

\section{ABSTRACTS}

L'objet de cet article est l'analyse d'un phénomène banal mais troublant: la confusion que l'énonciation d'un métatexte portant sur un texte de fiction crée entre le (monde du) texte et le (monde du) métatexte, entre (énoncé) fictif et (commentaire) réel. Cette confusion énonciative réalise une transgression de même nature que celle qu'opère la métalepse narrative, mais c'est le lecteur qui, dans son commentaire, en est le responsable : c'est pourquoi l'on peut parler d'une métalepse du lecteur. L'article propose une définition de cette métalepse du lecteur en traçant sa généalogie théorique, puis ouvre des perspectives en montrant comment la métalepse du lecteur est liée à toute lecture de textes de fiction et en identifiant certaines de ses caractéristiques qui sont communes à tout discours métatextuel.

\section{INDEX}

Mots-clés: compréhension, détextualité, évaluation, fiction et réalité, métalepse du lecteur, métaphrase, métatexte, paraphrase, PISA, texte littéraire

\section{AUTHOR}

\section{BERTRAND DAUNAY}

Université de Lille, Théodile-CIREL (EA 4354)

Bertrand Daunay est professeur de didactique à l'université de Lille dans le Nord de la France, membre de l'équipe de recherche Théodile-CIREL - EA 4354 (dont il a été le directeur de 2008 à 
2013), président d'honneur de l'AIRDF (après en avoir été président de 2010-2016).

Ses activités de recherche portent sur la didactique du français, avec une focalisation sur

l'approche didactique de la littérature, et le conduisent à interroger les fondements

épistémologiques et méthodologiques de la didactique du français et plus généralement des didactiques des disciplines.

Parmi ses dernières publications, signalons celles qui ont été réalisées avec Daniel Bart autour du programme international d'évaluation des élèves, le PISA, dont l'ouvrage paru aux éditions du Croquant en 2016, Les Blagues à PISA. 